\title{
Evidence of quasi-chemically homogeneous evolution of massive stars up to solar metallicity ${ }^{\star}$
}

\author{
F. Martins ${ }^{1}$, E. Depagne ${ }^{2}$, D. Russeil ${ }^{3}$, and L. Mahy ${ }^{4}$ \\ 1 LUPM-UMR 5299, CNRS \& Université Montpellier II, Place Eugène Bataillon, 34095 Montpellier Cedex 05, France \\ e-mail: fabrice.martins@univ-montp2.fr \\ ${ }^{2}$ Leibniz-Institut für Astrophysik Potsdam, An der Sternwarte 16, 14482 Potsdam, Germany \\ 3 Aix Marseille Université, CNRS, LAM (Laboratoire d'Astrophysique de Marseille) UMR 7326, 13388 Marseille, France \\ ${ }^{4}$ Institut d'Astrophysique et de Géophysique, Université de Liège, Bât B5C, Allée du 6 Août 17, 4000 Liège, Belgium
}

Received 12 February 2013 / Accepted 9 April 2013

\begin{abstract}
Context. Long soft gamma-ray bursts (LGRBs) are usually associated with the death of the most massive stars. A large amount of core angular momentum in the phases preceding the explosion is required to form LGRBs. A very high initial rotational velocity can provide this angular momentum. This velocity strongly influences the way the star evolves: it is mixed in a chemically homogeneous way and evolves directly towards the blue part of the Hertzsprung-Russell (HR) diagram from the main sequence.

Aims. We have shown that chemically homogeneous evolution takes place in the Small Magellanic Cloud (SMC) at low metallicity. We want to see whether there is a metallicity threshold above which such an evolution is not possible.

Methods. We performed a spectroscopic analysis of H-rich early-type WN stars in the Large Magellanic Cloud (LMC) and the Galaxy. We used the code CMFGEN to determine the fundamental properties $\left(T_{\text {eff }}, L\right)$ and the surface composition of the target stars. We then placed the stars in the HR diagram and determined their evolution.

Results. We show that both the LMC and Galactic WNh stars we selected cannot be explained by standard stellar evolution. They are located on the left of the main sequence but show surface abundances typical of $\mathrm{CN}$ equilibrium. In addition, they still contain a large amount of hydrogen. They are thus core-H burning objects. Their properties are consistent with chemically homogeneous evolution. We determine the metallicity of the Galactic stars from their position and Galactic metallicity gradients and conclude that they have $0.6<Z<1$.0. A moderate coupling between the core and the envelope is required to explain that stellar winds do not extract too much angular momentum to prevent a blueward evolution.

Conclusions. We have shown that chemically homogeneous evolution takes place in environments with metallicity up to solar. Since some long gamma-ray bursts appear in (super-)solar environments, such an evolution may be a viable way to form them over a wide range of metallicities.
\end{abstract}

Key words. stars: massive - stars: Wolf-Rayet - stars: evolution - stars: early-type

\section{Introduction}

Long soft gamma-ray bursts (LGRB) are classically associated with the death of massive stars. The most convincing evidence for this connection is the clear association of some LGRBs with type Ib/c supernovae (Galama et al. 1998; Hjorth et al. 2003). They are also observed in star-forming galaxies, directly in the sites where stars are currently being formed (but see Hammer et al. 2006). The conditions necessary to produce LGRBs require that their progenitors have an envelope free of hydrogen, so the jet can travel through them without being damped. The progenitor core must also retain a large angular momentum in order to produce a disk-jet structure around the black hole, which are the results of the core collapse. The collapsar model proposed by Woosley et al. (1993) fulfills these conditions and is presently the main scenario used to explain the existence of LGRBs.

The conditions required by the collapsar scenario, especially the large angular momentum of the core before explosion, have implications for the properties of the progenitor star. Since massive stars lose mass at a high rate due to their strong radiatively

^ Figures 1-6 are available in electronic form at http://www . aanda.org driven stellar winds (Castor et al. 1975; Puls et al. 2008), they also lose a significant amount of angular momentum during their lives. However, massive stars winds also depend on metallicity $\left(\dot{M} \propto Z^{0.6-0.8}\right.$ Mokiem et al. 2007; Vink et al. 2001): the lower the metal content, the weaker the radiative acceleration through lines, and thus the lower the mass loss rate. The observation of higher rotational velocities in the LMC/SMC compared to the Galaxy (Martayan et al. 2006; Mokiem et al. 2006) is an indirect confirmation of this effect (although the initial distribution of rotational velocities might also be different). Massive stars in low-Z environments are thus more likely to become LGRBs in the scenario where the angular momentum evolution is governed by mass loss (see Petrovic et al. 2005b for alternative possibilities). An unusually large initial rotational velocity is also required, since not all low-Z massive stars retain enough angular momentum at the end of their life to become an LGRB.

Rapid rotation affects stellar evolution. Maeder (1987, see also Langer 1992) shows that the mixing timescale becomes shorter than the nuclear timescale for very fast rotating massive stars. In that case, the material produced in the stellar core is immediately mixed with the outer layers. The star is thus (quasi-) chemically homogeneous. The larger fraction of helium in the outer layers reduces the opacity, causing the star 
Table 1. Sample stars and observational information.

\begin{tabular}{|c|c|c|c|c|c|c|}
\hline Star & Optical spectrum & Date of optical observation & Resolution & UV spectrum & Date of UV observation & Resolution \\
\hline \multicolumn{7}{|l|}{ Galaxy } \\
\hline WR 7 & VLT/UVES & 26-27 Jan. 2008 & 30000 & IUE sp03541 & 6 Dec. 1978 & 250 \\
\hline WR 10 & VLT/UVES & 26-27 Jan. 2008 & 30000 & IUE sp10748/sp13912 & 4 Dec. $1980 / 6$ May 1981 & 250 \\
\hline WR 18 & VLT/UVES & 26-27 Jan. 2008 & 30000 & IUE sp0933 & 18 May 1980 & 250 \\
\hline WR 128 & NTT/FEROS & 3 May 2012 & 48000 & IUE sp15101 & 26 Sep. 1981 & 18000 \\
\hline \multicolumn{7}{|l|}{ LMC } \\
\hline Bat 18 & $\mathrm{SAAO} / \mathrm{GS}^{1}$ & $\begin{array}{c}\text { 28 Dec. } 1999 \\
\text { 16-20 Jan. } 2001 \\
\text { 1-7 Jan. } 2002\end{array}$ & 900 & IUE sp38236/sp38247 & 24 Fev. 1990 & 250 \\
\hline Bat 63 & $\mathrm{SAAO} / \mathrm{GS}^{1}$ & $\begin{array}{l}28 \text { Dec. } 1999 \\
\text { 16-20 Jan. } 2001 \\
\text { 1-7 Jan. } 2002\end{array}$ & 900 & IUE sp09157/sp09158 & 31 May 1980 & 250 \\
\hline
\end{tabular}

Notes. ${ }^{(1)}$ SAAO/GS stands for SAAO observatory/Grating spectrograph. From Foellmi et al. (2003).

to become hotter. Consequently, chemically homogeneous stars evolve directly to the blue part of the HR diagram from the main sequence.

We showed that quasi-chemically homogeneous evolution (CHE in the following) was most likely followed by the star WR 1 in the SMC (Martins et al. 2009). The analysis of its UV-optical-IR spectrum indicated a hot temperature $(65000 \mathrm{~K})$, placing the star on the blue side of the main sequence in the HR diagram. At the same time, we determined that WR 1 still contained a large amount of hydrogen in its envelope. This was consistent with its spectral classification (WN5h) which is typical of WN stars that show the presence of hydrogen lines in their atmosphere. Classical evolutionary tracks are not able to reproduce both the position in the HR diagram and the large hydrogen content. When they reach the position of WR 1, they have long burned all of their hydrogen. Only CHE can explain the properties of WR 1. This is supported by our determination of the CNO content, which is fully consistent with the values expected from CNO equilibrium. SMC WR 1 is thus very likely to be a massive star in the core-H burning phase and it evolves homogeneously toward the blue part of the HR diagram. It validates the concept that some stars follow CHE even if this does not necessarily mean that they will end as an LGRB.

This study was focused on stars in the SMC. Whether CHE also happens at higher metallicity (see also Gräfener et al. 2011) is important in the context of the collapsar model. The first determinations of the metallicity of LGRB hosts tended to show that low- $Z$ environments were preferred. For instance, Modjaz et al. (2008) shows that type Ic supernovae associated with LGRBs were located in galaxies with metallicities that are systematically lower than type Ic SN without GRB. Various analysis of LGRB hosts confirmed that low metallicity was preferred (Thöne et al. 2008; Levesque et al. 2011). On the theoretical side, Yoon et al. (2006) shows that stellar evolution at metallicities above 0.6 ended with too low angular momentum in the core to produce LGRBs. A threshold in metallicity of around $0.6 Z_{\odot}$ for the formation of LGRBs seemed to exist.

With the discovery of at least two LGRBs at super-solar metallicity (Graham et al. 2009; Levesque et al. 2010), the observational side has seen some recent changes. The determination of the mass-metallicity relation for LGRB hosts indicates that solar and super-solar metallicities are not excluded, although the relation is shifted toward lower $Z$ compared to the classical $M-Z$ relation (Mannucci et al. 2011). The tendency of LGRBs to be located in actively star-forming galaxies explains this offset: these galaxies are currently mainly low-mass galaxies.

The question of CHE at high metallicity then arises. Massive stars never evolving homogeneously at high $Z$ might favor alternative evolutionary paths to form LGRBs in high metallicity environments. Petrovic et al. (2005b; see also Gräfener et al. 2012) shows that the accreting star of a close binary, or a single star, might retain enough angular momentum after the red supergiant phase to produce a LGRB, under the assumption of a weak coupling between the core and the envelope. In the present paper, we report on the analysis of hydrogen-rich WN stars in the LMC and the Galaxy. In a manner similar to the WNh stars in the SMC, we determine their physical properties and conclude about their evolution. We show that CHE is likely to exist in the LMC and the Galaxy, at solar metallicity.

The paper is organized as follows: in Sect. 2 we describe our sample and the observations; we then explain how we determined the fundamental properties of the sample stars in Sect. 3; we present our results in Sect. 4 and we discuss them in Sect. 5. The conclusions are gathered in Sect. 6.

\section{Sample stars and observations}

The optical data have been obtained from a variety of telescopes/instruments. Data for the LMC stars have been collected at SAAO and are those of Foellmi et al. (2003). Details of the data reduction can be found in Foellmi et al. (2003). We have used the average of all optical spectra in our analysis to provide average stellar parameters. The optical spectrum of WR 128 was collected by one of us (LM) during run 089.D-0730(A) on the $2.2 \mathrm{~m}$ telescope that is equipped with the FEROS spectrograph at the La Silla ESO observatory. The reduction procedure is identical to that described in Sana et al. (2006) and was done under the MIDAS environment. Data for WR 7, WR 18 and WR 128 were obtained with the spectrograph UVES on ESO/VLT (run 080.D-0137(A)). UVES spectra were reduced using the UVES pipeline provided by ESO, which performs bias and inter-order background subtraction (object and flat-field), optimal extraction of the object (above sky, rejecting cosmic ray hits), division by a flat-field frame extracted with the same weighted profile as the object, wavelength calibration and rebinning to a constant wavelength and step, and merging of all overlapping orders. In addition to the optical spectra, we have collected UV data from the IUE archive. Information on the spectra are given in Table 1. 
Table 2. Photometry and distance of the target stars.

\begin{tabular}{llrrrrrrr}
\hline \hline Star & ST & $U$ & $B$ & $V$ & $J$ & $H$ & $K$ & $\begin{array}{r}\text { distance } \\
{[\mathrm{kpc}]}\end{array}$ \\
\hline Galaxy & & & & & & & & \\
\hline WR 7 & WN4 & 11.21 & 11.68 & 11.40 & 9.97 & 9.67 & 9.27 & 3.67 \\
WR 10 & WN5h & 10.62 & 11.30 & 10.90 & 10.05 & 9.89 & 9.61 & 4.68 \\
WR 18 & WN4 & 11.10 & 11.40 & 10.60 & 8.57 & 8.21 & 7.68 & 2.20 \\
WR 128 & WN4(h) & 9.59 & 10.54 & 10.46 & 9.97 & 9.84 & 9.62 & 9.37 \\
\hline LMC & & & & & & & & \\
\hline Bat 18 & WN3h & 13.57 & 14.45 & 14.67 & 14.63 & 14.49 & 14.40 & 50.12 \\
Bat 63 & WN4ha & 13.62 & 14.45 & 14.60 & 14.84 & 14.62 & 14.50 & 50.12 \\
\hline
\end{tabular}

Notes. References for the distances are: Massey et al. (2001) for WR10, van der Hucht (2001) for WR18, 7, 128. The distance modulus to the LMC is assumed to be 18.5 .

When several IUE spectra are listed, we used the average spectrum to perform our spectroscopic analysis.

\section{Modeling}

We have used the code CMFGEN (Hillier \& Miller 1998) to determine the stellar and wind parameters of the sample stars. CMFGEN solves the radiative transfer in the comoving frame in non-LTE conditions. A spherical geometry is adopted to correctly take the wind extension into account. The rate equations and the radiative equation are solved simultaneously through an iterative scheme until convergence of the level populations has been achieved. The temperature structure is set by the radiative equilibrium constraint. The velocity law (and consequently, the density law through the mass conservation equation) is the result of the combination of an inner structure connected to a $\beta$ velocity law, in the outer atmosphere. The inner structure is iterated: once the level populations have converged, the total radiative acceleration is calculated and the momentum equation is solved to provide a new velocity law. This solution is connected to the $\beta$ velocity law and the resulting velocity structure is used to compute a new atmosphere model with new level populations. A couple of these hydrodynamical simulations is performed. In the model computation, a constant microturbulent velocity of $50 \mathrm{~km} \mathrm{~s}^{-1}$ is adopted. The elements included in our models are $\mathrm{H}, \mathrm{He}, \mathrm{C}, \mathrm{N}$, $\mathrm{O}, \mathrm{Ne}, \mathrm{P}, \mathrm{Si}, \mathrm{S}, \mathrm{Fe}, \mathrm{Ni}$. We use the solar abundances of Grevesse et al. (2007) as a reference.

Once the atmosphere model is completed, a formal solution of the radiative transfer equation is performed to yield the emergent spectrum. A depth variable microturbulence velocity from $10 \mathrm{~km} \mathrm{~s}^{-1}$ at the bottom of the wind to $10 \%$ of the terminal velocity in the outter layers is adopted. The emergent spectrum is subsequently compared to the observed data to determine the stellar and wind parameters. In practice, we proceed as follows:

- Effective temperature: we use the ionization balance method to constrain $T_{\text {eff. }}$. The ratio of N IV to $\mathrm{N} \mathrm{V}$ lines is a first indicator. N IV 4058, N IV 5205, N IV 5736, N IV 7103, $\mathrm{N} \mathrm{V}$ 4519-4523 and N v 4944 are the main indicators. We also use the strength of the He II lines and, when observed, of He I 5876 as a secondary temperature indicators. The Fe V 1350-1400 forest in the UV range provides an additional constraint. For Wolf-Rayet stars, a meaningful quantity is the temperature at an optical depth of about $20\left(T_{*}\right)$. This quantity is appropriate to compare with temperatures provided by evolutionary models, since it corresponds to the quasi-hydrostatic part of the atmosphere.

- Luminosity: the luminosity is derived from the fit of the spectral energy distribution (SED). Flux-calibrated IUE spectra and $U B V J H K$ photometry are used to constrain $\log \frac{L}{L_{\odot}}$ and the amount of extinction. The distances we adopted are listed in Table 2. For the Galactic objects, they rely on the correlation between the position of the target stars and OB associations. Due to uncertainties in these correlations and in the distance of Galactic associations, the errors on the luminosity of the Galactic WNh stars might be underestimated.

- Mass loss rate: it is derived from the absolute strength of the emission lines. $\mathrm{H} \alpha, \mathrm{H} \beta, \mathrm{H} \gamma$ and $\mathrm{He}$ II 4686 are the main indicators. The UV resonance lines (N V 1240, C IV 1550, He II 1640, N IV 1720) are also used. Generally, the optical and UV wind-sensitive lines give consistent results.

- Terminal velocity: the blueward extent of the UV resonance lines is the main indicator. The width of optical lines is the secondary diagnostic.

- Surface abundances: the He/H ratio is constrained from the relative strength of $\mathrm{H}$ and $\mathrm{He}$ II lines. This is different from the determination of the mass loss rate in which the absolute level of emission is used. The blends $\mathrm{He} \mathrm{II} / \mathrm{H} \gamma$ and $\mathrm{He} \mathrm{II} / \mathrm{H} \beta$, and the single lines He II 4542 and He II 5412 are the main indicators. Carbon abundances are determined from C IV 5802-5812. Nitrogen abundances result from the analysis of N IV 4058, N V 4519-23, N V 4944, N IV 5205, N IV 5735 and, when available, N IV 7103.

- Clumping factor: we used mainly the red wing of the strong optical emission lines to determine the volume filling factor $f$ (in the micro-clumping formalism). According to Hillier (1991), the electron scattering wing depends on the degree of inhomogeneity, which is stronger in homogeneous winds. In practice, He II 4686 provides the best constraints.

- Slope of the velocity field: we have used a value of 1.0 for the so-called $\beta$ parameter in our modelling of the Galactic targets. It provided a good fit of the Balmer and He II emission lines. For the LMC stars, we found that $\beta=2.0$ (respectively 1.5 ) leads to a better shape of the optical emission lines of Bat 18 (respectively Bat 63).

The typical uncertainties have been estimated by varying the main parameters around the preferred values. When a clear deviation is observed in the fit, we assume we are seeing the effect of a one-sigma deviation from the best set of parameters. The uncertainties are the following: $3000 \mathrm{~K}$ on the temperature; $0.10-0.15$ dex on the luminosity, 0.2 dex on the mass loss rates, $200 \mathrm{~km} \mathrm{~s}^{-1}$ on the terminal velocity and 30 to $40 \%$ on the surface abundances.

\section{Results}

\subsection{Stellar parameters}

The best fit to the observed spectra of our sample stars are shown in Figs. 1 to 6 . They are usually of excellent quality. Most 
Table 3. Parameters of the target stars determined from spectroscopic analysis.

\begin{tabular}{|c|c|c|c|c|c|c|c|c|c|c|c|c|}
\hline Star & ST & $\begin{array}{r}T_{\text {eff }} \\
{[\mathrm{kK}]}\end{array}$ & $\begin{array}{r}T_{*} \\
{[\mathrm{kK}]}\end{array}$ & $\log \frac{L}{L_{\odot}}$ & $\begin{array}{r}R_{*} \\
{\left[R_{\odot}\right]}\end{array}$ & $\log (\dot{M})$ & $\begin{array}{r}v_{\infty} \\
{\left[\mathrm{km} \mathrm{s}^{-1}\right]}\end{array}$ & $f$ & $X(\mathrm{H})$ & $\mathrm{He} / \mathrm{H}$ & $\begin{array}{r}X(\mathrm{C}) \\
{\left[\times 10^{-5}\right]}\end{array}$ & $\begin{array}{r}X(\mathrm{~N}) \\
{\left[\times 10^{-3}\right]}\end{array}$ \\
\hline \multicolumn{13}{|l|}{ Galaxy } \\
\hline WR 7 & WN4 & 60.0 & 80.8 & 5.40 & 2.57 & -4.80 & 1600 & 0.1 & $<0.01$ & $>10$ & $14.7 \pm 5.0$ & $11.0 \pm 5.0$ \\
\hline WR 10 & WN5h & 53.5 & 55.2 & 5.45 & 5.79 & $-5.40 /-5.45$ & 1400 & 0.1 & $0.33 \pm 0.11$ & $0.50 \pm 0.20$ & $7.9 \pm 3.0$ & $14.0 \pm 5.0$ \\
\hline WR 18 & WN4 & 56.0 & 74.1 & 5.30 & 2.73 & -4.60 & 2200 & 0.3 & $<0.01$ & $>10$ & $17.7 \pm 5.0$ & $10.3 \pm 4.0$ \\
\hline WR 128 & WN4(h) & 57.0 & 59.9 & 5.50 & 5.43 & -5.30 & 1800 & 0.1 & $0.26 \pm 0.07$ & $0.70 \pm 0.20$ & $6.2 \pm 1.6$ & $11.0 \pm 3.0$ \\
\hline \multicolumn{13}{|l|}{ LMC } \\
\hline Bat 18 & WN3h & 60.0 & 72.8 & 5.50 & 3.54 & -5.02 & 1800 & 0.3 & $0.25 \pm 0.10$ & $0.80 \pm 0.20$ & $5.7 \pm 2.0$ & $6.6 \pm 2.1$ \\
\hline Bat 63 & WN4ha & 58.5 & 68.9 & 5.45 & 3.73 & -5.45 & 2000 & 0.1 & $0.40 \pm 0.20$ & $0.35 \pm 0.15$ & $20.0 \pm 15.0$ & $2.3 \pm 1.6$ \\
\hline
\end{tabular}
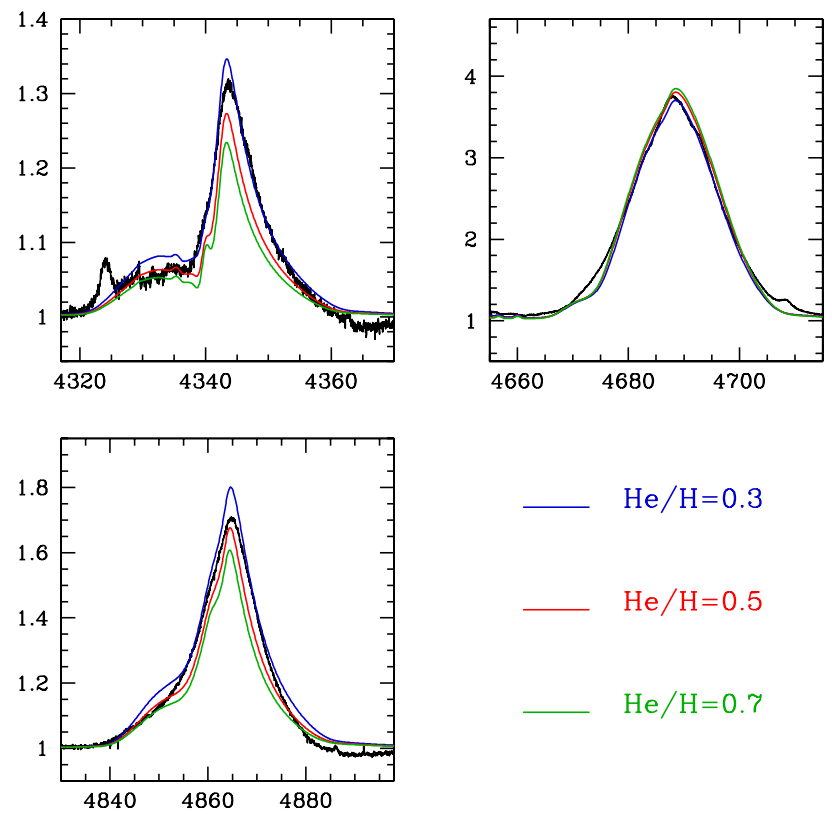

Fig. 7. Comparison between the observed spectrum of WR 10 (black line) and CMFGEN models with different $\mathrm{He} / \mathrm{H}$ ratios. The lines are $\mathrm{H} \beta$ (upper left), HeII 4686 (upper right), and $\mathrm{H} \gamma$ (lower left). The mass loss rate of the models is adjusted, so that HeII 4686 is always correctly reproduced.

lines are well reproduced. We note a difficulty to reproduce the N v 4605-4620 features in Galactic stars. They are usually too weak when compared to the observed spectrum. The other nitrogen lines are well reproduced, especially those used to determine the effective temperature. The reason for this discrepancy is not known at present. Similar problems were noted by Bouret et al. (2012). The derived parameters are gathered in Table 3.

One of the key parameters that we obtained is the hydrogen content of the stars. In Fig. 7 we illustrate the determination of the $\mathrm{He} / \mathrm{H}$ ratio in the case of star WR 10 . The models all reproduce correctly the $\mathrm{He}$ II 4686 emission. For $\mathrm{He} / \mathrm{H}=0.3$, the hydrogen content is too large and the Balmer lines are too strong. Alternatively, for $\mathrm{He} / \mathrm{H}=0.7$, the hydrogen content is too small and the Balmer emission is too weak. Consequently, a value of $\mathrm{He} / \mathrm{H}=0.5$ is preferred. This also gives an estimate of the uncertainty on the $\mathrm{He} / \mathrm{H}$ determination. We see that all WNh stars have a non negligible fraction of hydrogen in their atmosphere, while the two comparison WN4 stars are hydrogen-free.

The four Galactic stars were analyzed by Hamann et al. (2006). We usually derive lower temperatures. The difference is about $10000 \mathrm{~K}$ for WR 10 and WR 128 but amounts to $20000-30000 \mathrm{~K}$ for WR 7 and WR 18 . It is difficult to significantly increase the temperature of our models for those objects, since the Fe V forest at around 1300-1400 $\AA$ becomes very weak, contrary to what is observed. On the other hand, $\mathrm{N} \mathrm{v} \mathrm{4605-4620} \mathrm{is} \mathrm{better} \mathrm{reproduced} \mathrm{at} \mathrm{such} \mathrm{high} \mathrm{temperatures}$ (see also Bouret et al. 2012).

The luminosities that we derive for the Galactic stars are consistent with the results of Hamann et al. (2006). Our mass loss rate determination is also in good agreement: differences are smaller than 0.2 dex. Hamann et al. (2006) did not find any evidence for the presence of hydrogen in WR 7 and WR 18, which is consistent with our determinations. For WR 10 and WR 128, they quote a hydrogen mass fraction of 0.25 and 0.16 , respectively. This is again in good agreement with our results.

\subsection{Evolutionary status}

The location of the sample stars in the HR diagram is shown in Fig. 8. The evolutionary tracks are from Geneva and include rotation. Two sets of tracks are displayed: the ones by Meynet \& Maeder (2005) (left) and the ones by Ekström et al. (2012) (right). The solar metallicity reference is $Z=0.020$ in the Meynet \& Maeder tracks, while it is $Z=0.014$ in the Ekström et al. tracks. We show in bold the part of the evolutionary tracks for which the hydrogen mass fraction is higher than 0.1 . This value is lower than the values we determine for all WNh stars of our sample. The Galactic stars cannot be reproduced by the 2005 tracks. Their position in the HR diagram is reached only by hydrogen-free tracks. With the more recent tracks, the stars are at the limit of the region reached by $\sim 25 M_{\odot}$ track which still contains a non-zero fraction of hydrogen. They can be only marginally represented by those tracks. LMC tracks with normal $\left(300 \mathrm{~km} \mathrm{~s}^{-1}\right)$ and fast $\left(550 \mathrm{~km} \mathrm{~s}^{-1}\right)$ rotation are used to build the HR diagram shown in Fig. 9. The two LMC stars are clearly too hot to be reproduced by standard evolutionary tracks which never come back to the blue part of the HR diagram with a significant amount of hydrogen at their surface, in contrast to what Bat 18 and Bat 63 show. From those comparisons, one can conclude that the LMC WNh stars and very likely the Galactic ones cannot be explained by standard evolutionary tracks. This is similar to what we obtained for the SMC stars (Martins et al. 2009).

In this study, we concluded that $\mathrm{CHE}$ could be a way to explain the properties of the SMC early-type WNh stars. This evolution takes place when the mixing timescale becomes shorter than the nuclear timescale. This is possible if the stars rotate initially very fast and do not lose too much angular momentum during their evolution. The Geneva tracks used so far exist only for an initial rotational velocity of 0 or $300 \mathrm{~km} \mathrm{~s}^{-1}$. In Fig. 9 , we show the HR diagram that was built with the evolutionary tracks of Brott et al. (2011) using a LMC composition. Those tracks have been computed for a variety of rotational velocities. 


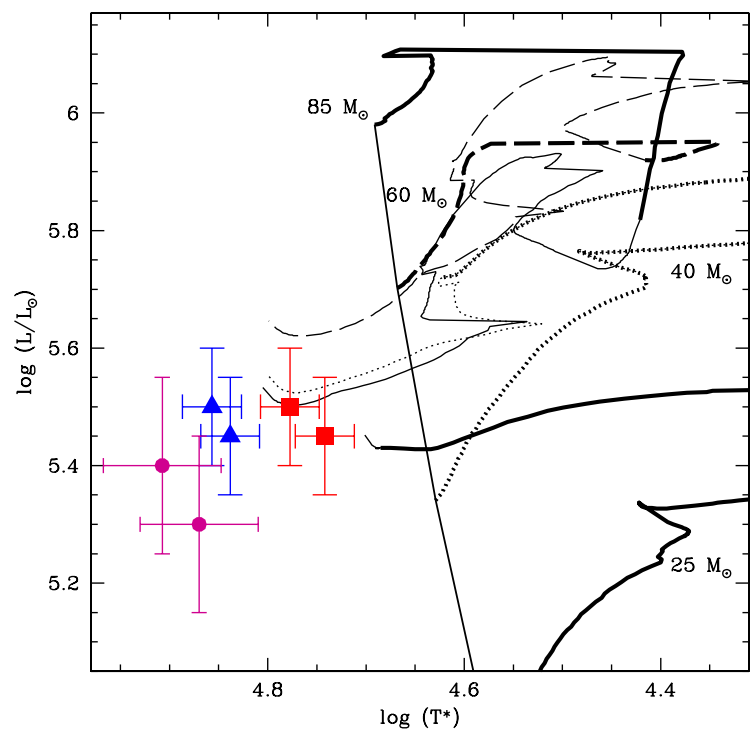

(a)

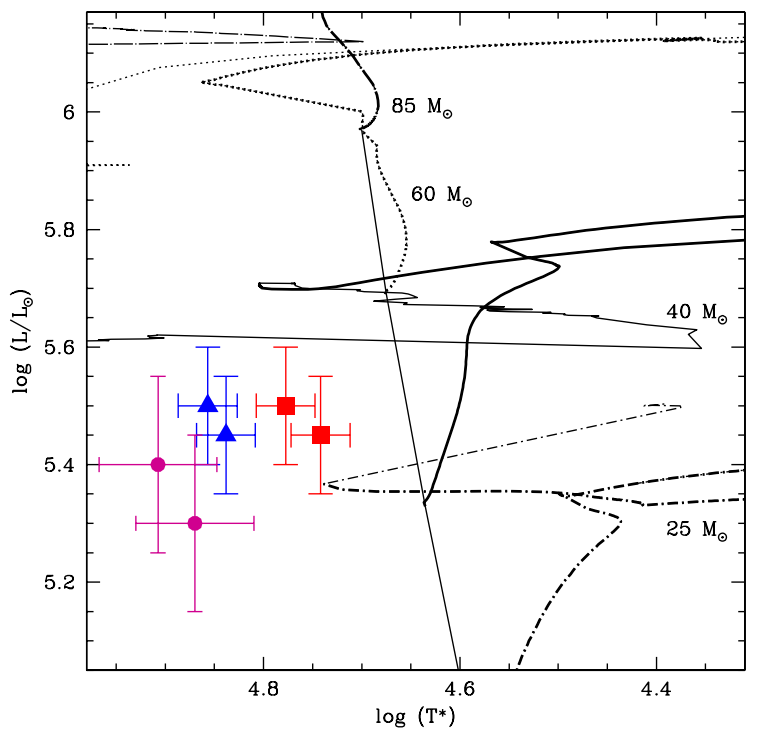

(b)

Fig. 8. HR diagram with the position of the sample WN stars shown by triangles (for LMC targets) and squares (Galactic targets). Circles correspond to the comparison Galactic WN4 stars. On the left panel, the evolutionary tracks are from Meynet \& Maeder (2005), while on the right panel they are from Ekström et al. (2012). The bold part of the tracks corresponds to a hydrogen mass fraction higher than 0.1.

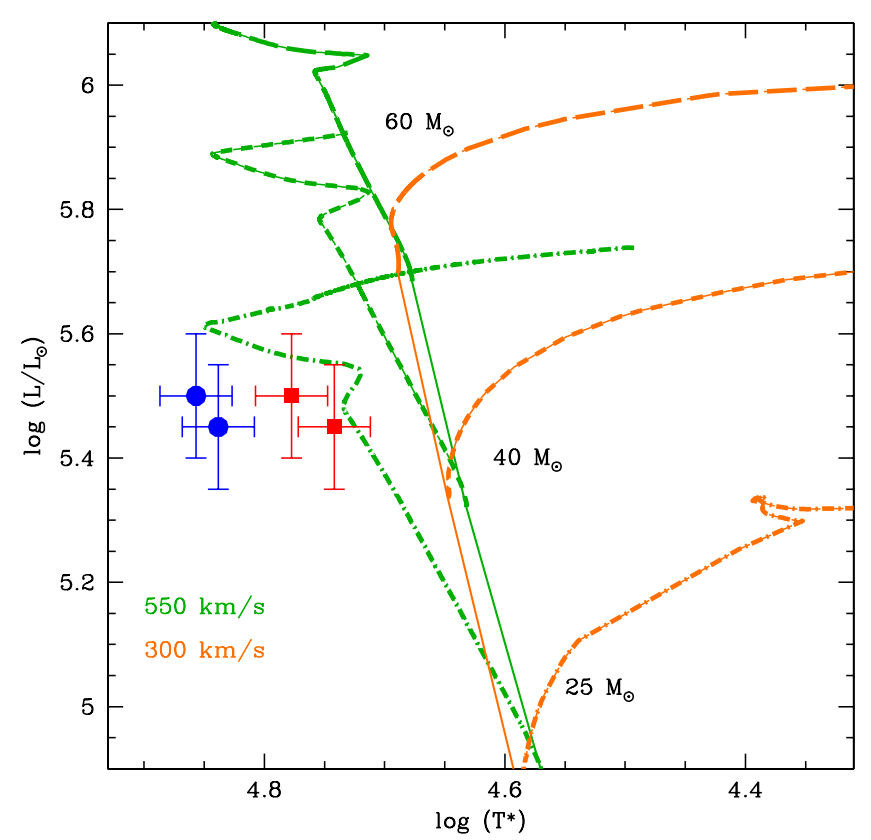

Fig. 9. HR diagram with the evolutionary tracks of Brott et al. (2011) with an initial rotational velocity of $300 \mathrm{~km} \mathrm{~s}^{-1}$ (orange) and $550 \mathrm{~km} \mathrm{~s}^{-1}$ (green). The symbols have the same meaning as in Fig. 8. The tracks all correspond to $\mathrm{He} / \mathrm{H}<2.0$ (equivalent of $X(\mathrm{H}) \gtrsim 0.1$ ).

We have plotted the tracks with initial velocities of 300 and $550 \mathrm{~km} \mathrm{~s}^{-1}$. The latter tracks evolve directly to the blue part of the HR diagram from the main sequence. This is very different compared to the $300 \mathrm{~km} \mathrm{~s}^{-1}$ tracks. The positions of the stars are well reproduced by the fast-rotating tracks with an initial mass close to $25 M_{\odot}$. In addition, they have $X(\mathrm{H})>0.1$, which is consistent with our spectroscopic analysis. We can thus conclude that the sample stars (at least those from the LMC) are consistent with a CHE. Bestenlehner et al. (2011) studied the WN5h LMC star VFTS682 and reached the same conclusion from its position in the HR diagram (they did not constrain the surface abundances).

Brott et al. (2011) have shown that they could produce this type of blueward evolution only at metallicities below that of the LMC. Their fast-rotating solar metallicity tracks behave more classically with a redward extension from the main sequence. If we look at Fig. 8, we see that the recent Geneva tracks at solar metallicity tend to evolve towards the blue rapidly after the main sequence. The 2005 Geneva models used $Z=0.020$, while the 2012 tracks use the revised solar abundances, which correspond to $Z=0.014$. This might favor chemically homogeneous evolution at high rotation. This leaves room for a pure homogeneous evolution for higher initial velocities even at solar metallicity.

In Fig. 10 we show the carbon mass fraction as a function of nitrogen mass fraction for our target stars. The rotating tracks from Meynet \& Maeder (2005) and Ekström et al. (2012) are overplotted. The WNh stars are located in the part of the diagram which corresponds to the minimum of carbon content and the maximum of nitrogen content (for their respective metallicity). This is typical of stars that show products of the $\mathrm{CN}$ cycle at their surface. This is a very strong indication that the WNh stars are core H-burning objects. If the stars evolve in a quasi-chemically homogeneous way and if they show $\mathrm{CN}$ equilibrium surface abundances, this means that their core $\mathrm{CN}$ abundances are also at equilibrium. Thus, the stars are still in the core H-burning phase. If they were more evolved, the central $\mathrm{CN}$ abundances would be no longer those at $\mathrm{CN}$ equilibrium.

The properties of the sample early-type WNh stars are similar to the SMC WNh stars that are studied by Martins et al. (2009). They are relatively unevolved objects. WR 7 and WR 18, the compared Galactic stars, are as nitrogen rich as the two Galactic WNh stars but show a significantly larger carbon content. This is convincing evidence that they are in a more advanced state of evolution since evolutionary models predict that the carbon mass fraction starts to increase due to the onset of core He-burning after reaching a minimum. These Galactic WN stars certainly follow normal evolution and have experienced a classical redward evolution in the HR diagram before 


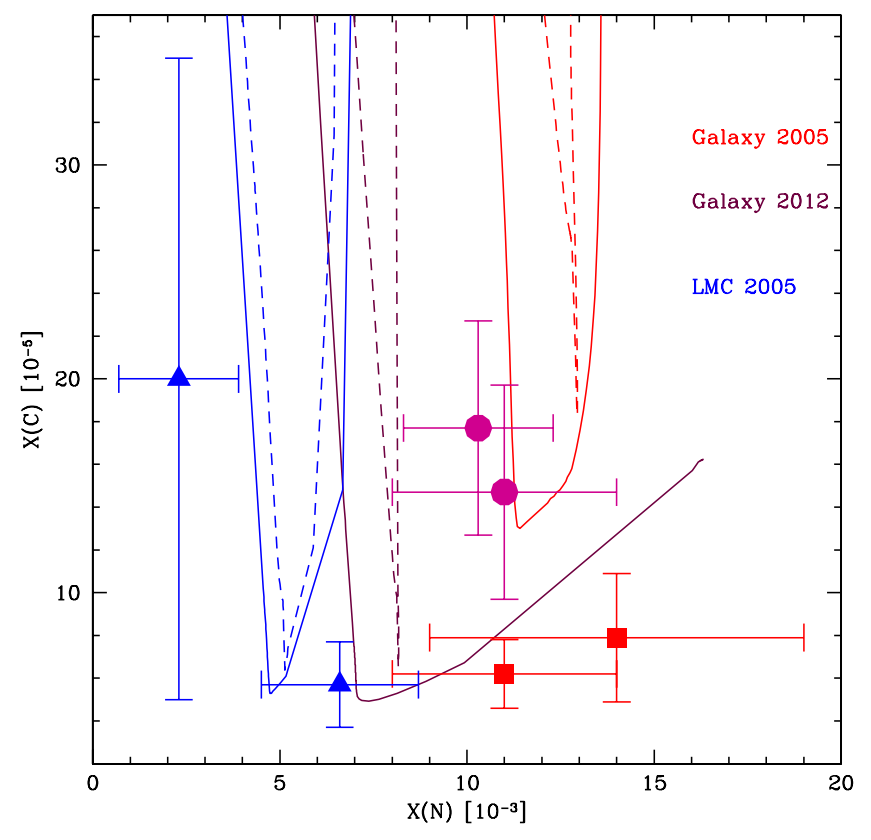

Fig. 10. Carbon mass fraction as a function of nitrogen mass fraction for the sample stars. The red squares (blue triangles) are the Galactic (LMC) objects. The Galactic compared stars are shown by the purple circles. Evolutionary tracks from Meynet \& Maeder (2005) at solar (LMC) metallicity are shown by red (blue) lines. The solar-metallicity tracks of Ekström et al. (2012) are shown by the dark red lines. Solid and dashed lines correspond respectively to 25 (30 at LMC metallicity) and $60 M_{\odot}$ stars.

coming back to the left of the ZAMS after having burned all of their hydrogen. The surface composition of WN stars is thus a key diagnostic of their type of evolution.

\section{Discussion}

Our results show that $\mathrm{CHE}$ takes place in galaxies with average metallicities higher than the SMC. Since metallicity gradients exist within galaxies, this does not necessarily mean that our sample stars are located in regions with large metal content. Although we have assumed that the LMC stars have a metal content 0.5 times the solar value, we have tried to obtain better estimates for the Galactic objects. We have used the position of the Galactic stars in the disk to estimate their metallicity from known $\mathrm{O} / \mathrm{H}$ and $\mathrm{Fe} / \mathrm{H}$ gradients which are established from Planetary Nebulae, HII regions, and cepheids. Planetary Nebulae and HII regions are two types of objects which are commonly used to determine the abundance gradient in the Milky Way disk. The oxygen abundance, which is easily observed in such emission-line objects, is expected to give the interstellar matter abundance at the time of the formation of the stars. Cepheids provide the iron content as a function of galactocentric radius (Lemasle et al. 2007). The results are given in Table 4. For HII regions (Balser et al. 2011), we provide two values: one from the azimuthally averaged gradient (Zhii) and one from the relation established in the azimuthal range which corresponds to the star's galactic longitude (Zhiib). For Planetary nebulae, we use the relation of Henry et al. (2010). The O/H and Fe/H determinations give consistent results. From Table 4, we clearly see that WR 128 has a near solar composition. WR 10 is located in a slightly less enriched medium, where $Z \sim 0.60-0.65$. With the assumption that the LMC targets have a metallicity of 0.5 the
Table 4. Metallicity estimates.

\begin{tabular}{lcccccc}
\hline \hline Star & $\begin{array}{c}d \\
{[\mathrm{kpc}]}\end{array}$ & $\begin{array}{c}R(\mathrm{kpc}) \\
{[\mathrm{kpc}]}\end{array}$ & Zpn & Zhii & Zhiib & Zcp \\
\hline WR128 & 4.30 & 7.03 & 1.05 & 0.97 & 1.07 & 1.13 \\
WR18 & 3.30 & 8.37 & 0.88 & 0.85 & 0.81 & 0.95 \\
WR10 & 4.60 & 11.19 & 0.60 & 0.64 & 0.65 & 0.66 \\
WR7 & 4.80 & 11.30 & 0.52 & 0.57 & 0.60 & 0.65 \\
\hline
\end{tabular}

Notes. $Z$ represents the metallicity. Zpn is obtained from from Planetary Nebulae; Zhii and Zhiib from HII regions (see text for discussion); Zcp from cepheids. It is expressed in oxygen abundance assuming $12+\log (\mathrm{O} / \mathrm{H})=8.66$ for the Sun (Grevesse et al. 2007) for planetary nebulae and HII regions, and in iron abundance for cepheids.

solar value, we conclude that the WNh stars which follow $\mathrm{CHE}$ are found in the interval $0.5-1.0 Z_{\odot}$.

We have not determined the metal content of each star directly from its spectrum. The Fe V and Fe VI lines in the UV range could be used in principle. However, only the Fe V forest around 1350-1400 $\AA$ is not dominated by strong emission lines. With only a single ionization state available, the determination of the Fe content is difficult since temperature uncertainties (approximately $3000 \mathrm{~K}$ ) strongly affect the estimate of metallicity. Thus, we think metallicity estimates that are based on galactic gradients are better than any direct diagnostic we have.

Our analysis provides good evidence of CHE at solar metallicity. The current evolutionary tracks at solar metallicity do not predict such an evolution (Brott et al. 2011; Ekström et al. 2012). Figure 5 of Brott et al. (2011) clearly shows that a blueward evolution for fast-rotating stars is possible only at metallicities below $0.5 Z_{\odot}$. This is usually interpreted as a wind effect. At higher metallicity, mass loss rates of massive stars are higher (Mokiem et al. 2007) and thus, the removal of angular momentum is easier. Stars brake faster and are never fully mixed, which is the necessary condition to have a homogeneous evolution. However, this interpretation assumes that the core and the envelope are strongly coupled, so that the reduction of the surface angular momentum by stellar wind is mirrored in the core angular momentum. If the coupling is weak or at least moderate, the effect of winds is reduced (see discussion in Georgy et al. 2012). The models of Brott et al. (2011) include the transport of angular momentum due to the presence of a magnetic field. This strengthens the core-envelope coupling, which partially explains that $\mathrm{CHE}$ is correlated with metallicity and wind strength. Petrovic et al. (2005b) also studied the angular momentum evolution of single and binary stars and concluded that the presence of magnetic field favored a slowly rotating core.

The solar-metallicity ( $Z=0.014)$ models of Ekström et al. (2012) which are seen in Fig. 8 (right), show slightly after the zero-age main sequence and above $40 M_{\odot}$ a clear deviation to the blue. These models do not include magnetic coupling. Qualitatively, they indicate that CHE is not excluded, since they are intermediate between fully chemically mixed models and models with normal mixing. Given the uncertainties in the mass loss rates and the physics of angular momentum transport, it is thus not excluded that evolutionary models can reproduce CHE.

According to the present results, the following conditions are required to reproduce the properties of the early WNh stars in the Galaxy: 1) a very efficient mixing of chemical species; 2) a fast initial rotation; and 3) a moderate or weak angular momentum coupling between the stellar core and the envelope. In such a way, the effect of stellar winds are reduced to the outer layers. 
This can explain that our WNh stars do not seem to be extremely fast rotators.

The determination of $V \sin i$ for our objects is very difficult since most lines are formed throughout the wind and are broadened by its expansion. Polarimetry sometimes indicates that Wolf-Rayet stars have a flattened shape which is probably due to rotation. Some of these objects may be identified as precursors of LGRBs (Vink et al. 2011; Gräfener et al. 2012). Even without polarimetric information, we can determine orders of magnitudes for the projected rotational velocity and clearly state that $V \sin i$ values larger than $400 \mathrm{~km} \mathrm{~s}^{-1}$ are safely excluded. Above such a value the details of some emission line profiles (such as C IV 5802-5812) are smoothed out. The surface projected velocities of our sample stars are thus of a few tens to a couple of hundreds of $\mathrm{km} \mathrm{s}^{-1}$ at most. Hence, a moderately low surface rotational velocity is not necessarily in contradiction with a CHE.

If the angular momentum coupling between the core and the envelope is only moderate, a star evolving homogeneously may form a collapsar (Woosley et al. 1993) and a long-soft gammaray burst. Recent observations reveal that such events are not restricted to low metallicity environments. Levesque et al. (2010) report a $L G R B$ in a galaxy with $12+\log (\mathrm{O} / \mathrm{H})=9.0$, which is larger than the solar value. Similarly, Mannucci et al. (2011) show that the mass-luminosity relation of LGRB hosts does not stop at solar metallicity: LGRB hosts are simply more massive for a given metallicity. Given the effects of stellar winds described above, it is likely that lower $Z$ favours CHE because braking is less efficient (even in the case of weak coupling, there is still an effect). But this type of evolution can also take place in solar-metallicity environments. The most recent compilation of Wolf-Rayet stars by Crowther ${ }^{1}$ contains 433 objects, among which only seven are of spectral type WN3h, WN4h or WN5h. If one assumes that such stars have similar properties to our sample stars, one may expect only 1-2\% of Wolf-Rayet stars to be massive stars that evolve quasi homogeneously. This is most likely an upper limit. Indeed, if WN3-5h stars are genuine core-H burning objects, their number should be compared to the number of normal main sequence stars, which is larger than the number of Wolf-Rayet stars. In the LMC, the catalog of Breysacher et al. (1999) lists 10 stars with spectral type WN3-5h among 134 objects, corresponding to a fraction of $7 \%$ of Wolf-Rayet stars being hydrogen-rich early-type WN objects. Even if a complete census of the Galactic Wolf-Rayet is still missing, it seems that the fraction of WN3-5h is larger in the LMC compared to the Galaxy. Assuming that all WN3-5h stars experience CHE, this is consistent with the theoretical prediction that $\mathrm{CHE}$ takes place more likely - but not exclusively - at low metallicity (Brott et al. 2011).

Fully mixed massive stars should be observed in the O-star phase when they are close to the zero-age main sequence. There are several indications that this is the case. Bouret et al. (2003) found that the hot O2III(f*) star MPG 355 in the SMC cluster NGC 346 is much younger than the bulk of $O$ stars (1 versus $3 \mathrm{Myr}$ ). They argue that it evolves homogeneously, which is consistent with its relatively high nitrogen enhancement triggered by fast rotation. Consequently, it follows isochrones much more "vertical" in the HR diagram and its age cannot be derived by standard isochrones. Similar conclusions are reached by Walborn et al. (2004) about ON2III stars. They are located close to the ZAMS in the HR diagram and feature a large N/C ratio. Mokiem et al. (2007) studied a sample of LMC stars and

\footnotetext{
1 http://pacrowther.staff.chef.ac.uk/WRcat/
}

reported a correlation between high helium enrichment and the presence of a mass discrepancy (difference between masses derived from spectroscopy and from the evolutionary tracks). A natural explanation is that the use of normally rotating tracks for stars that follow actually CHE will lead to a strong overestimate of the evolutionary mass. They thus conclude that some of the LMC O stars could be fully mixed. They also note that three of their $\mathrm{O} 2$ stars are located blueward of the main sequence. At least in the Magellanic Clouds, the indirect evidence of CHE in the $\mathrm{O}$ star phase are compelling. Similar results for Galactic O stars do not yet exist.

An alternative explanation for the position of the WNh stars in the HR diagram is binarity. As soon as the two components interact through mass transfer, evolutionary calculations of massive binary systems indicate that the evolutionary tracks are severely modified compared to single star tracks (Wellstein et al. 2001; Petrovic et al. 2005a): the accretor can turn back to the blue part of the HRD. Recent surveys of young star clusters indicate that 30 to $70 \%$ of massive stars have a companion (Sana et al. 2012, 2013). Mass transfer (and even merging) can occur during the early phases of evolution in short-period systems (de Mink et al. 2013). In such a case, the accreting star receives mass and angular momentum. It can appear bluer than the bulk of the surrounding population, similar to blue stragglers in globular clusters. This could explain the position of our sample WNh stars in the HR diagram. We have seen that the surface composition of the WNh stars was typical of $\mathrm{CN}$ equilibrium. In the binary scenario, the surface appearance of the accreting star strongly depends on the phase at which mass transfer occurs. If it is during the expansion of the primary, which is at the end of central hydrogen burning, the material dropped onto the secondary can show the signatures of $\mathrm{CN}$ processing. Depending on the initial period of the binary system, mass transfer can occur either before or much after hydrogen core exhaustion. In that case, no special surface abundance pattern is expected. It appears unlikely that binarity can explain the properties of all the earlytype WNh stars we have studied. Foellmi et al. (2003) studied the binary status of Bat 18 and Bat 63 and found no evidence of radial velocity nor photometric variations, which is consistent with them being single stars. No clear evidence of binarity exists for WR 10 and WR 128 either. We thus conclude that binarity could in principle explain the properties of some early-type WNh stars, but the ones we studied here are better accounted for by CHE.

All in all, there are now several robust indications that $\mathrm{CHE}$ happens at different metallicities, up to solar. An understanding of the conditions under which it appears is necessary since the current evolutionary models do not predict it at high metallicity. Beyond the understanding of a limited number of objects, their study is of importance to better constrain the mixing mechanism in massive stars. With better predictions, it will be easier to interpret data of LGRBs in various environments.

\section{Conclusion}

We have presented the analysis of early-type H-rich WN stars in the LMC and the Galaxy. The stellar and wind parameters have been determined through spectroscopic analysis in the UV and optical range. Atmosphere models and synthetic spectra computed with the code CMFGEN have been used. The main results are:

- the early-type WNh stars are hot objects located on the blue part of the zero age main sequence; 
- in spite of their Wolf-Rayet classification, they still contain a significant amount of hydrogen $(X(\mathrm{H})>0.2)$. In addition, their $\mathrm{C}$ and $\mathrm{N}$ composition is typical of the $\mathrm{CN}$ equilibrium. This indicates that the WN3-5h stars are corehydrogen burning objects. Comparison of $\mathrm{H}$-free WN4 stars indicates clear differences in the evolutionary state in both types of objects (WNh versus WN);

- classical evolutionary tracks cannot reproduce both the position in the HR diagram and the chemical composition of these objects. Fully mixed models, evolving blueward from the zero-age main sequence, are consistent with such properties. WN3-5h stars are most likely to follow CHE;

- estimates of the metallicity in the direct environment of the target stars reveals $0.5<Z<1.0$, indicating that CHE happens at solar metallicity. This favours a relatively moderate coupling between the core and the envelope for angular momentum transport, since the effect of stronger stellar winds at higher metallicity does not prevent $\mathrm{CHE}$, contrary to the current predictions of evolutionary models. This result is also consistent with the "not extreme" rotational velocity of the WN3-5h stars;

- the finding that CHE takes place in solar-metallicity environments and the discovery of LGRBs in metal-rich galaxies support the scenario where the progenitors of LGRBs stem from CHE.

The total number of WN3-5h stars in the Galaxy is relatively small (1-2\% of all Wolf-Rayet stars). If all WN3-5h stars follow CHE, this shows that this type of evolution is rare. It is however crucial to understand it - not only in the context of LGBR production but also to better constrain the physical processes in stellar interior, especially with regard to mixing and angular momentum transport. Future theoretical studies should help improve our knowledge of such effects.

Acknowledgements. We thank an anonymous referee for comments which helped to improve the clarity of our statements. We thank John Hillier for making his code CMFGEN available and for constant help with it. F.M. acknowledges support from the "Agence Nationale de la Recherche (ANR)". We thank Cédric Foellmi for providing us with the optical spectra of stars Bat 18 and Bat 63 .

\section{References}

Balser, D. S., Rood, R. T., Bania, T. M., \& Anderson, L. D. 2011, ApJ, 738, 27 Bestenlehner, J. M., Vink, J. S., Gräfener, G., et al. 2011, A\&A, 530, L14
Bouret, J.-C., Lanz, T., Hillier, D. J., et al. 2003, ApJ, 595, 1182

Bouret, J.-C., Hillier, D. J., Lanz, T., \& Fullerton, A. W. 2012, A\&A, 544, A67

Breysacher, J., Azzopardi, M., \& Testor, G. 1999, A\&AS, 137, 117

Brott, I., de Mink, S. E., Cantiello, M., et al. 2011, A\&A, 530, A115

Castor, J. I., Abbott, D. C., \& Klein, R. I. 1975, ApJ, 195, 157

de Mink, S. E., Langer, N., Izzard, R. G., Sana, H., \& de Koter, A. 2013, ApJ, 764,166

Ekström, S., Georgy, C., Eggenberger, P., et al. 2012, A\&A, 537, A146

Foellmi, C., Moffat, A. F. J., \& Guerrero, M. A. 2003, MNRAS, 338, 1025

Galama, T. J., Vreeswijk, P. M., van Paradijs, J., et al. 1998, Nature, 395, 670

Georgy, C., Ekström, S., Meynet, G., et al. 2012, A\&A, 542, A29

Gräfener, G., Vink, J. S., de Koter, A., \& Langer, N. 2011, A\&A, 535, A56

Gräfener, G., Vink, J. S., Harries, T. J., \& Langer, N. 2012, A\&A, 547, A83

Graham, J. F., Fruchter, A. S., Kewley, L. J., et al. 2009, in AIP Conf. Ser. 1133, eds. C. Meegan, C. Kouveliotou, \& N. Gehrels, 269

Grevesse, N., Asplund, M., \& Sauval, A. J. 2007, Space Sci. Rev., 130, 105

Hamann, W.-R., Gräfener, G., \& Liermann, A. 2006, A\&A, 457, 1015

Hammer, F., Flores, H., Schaerer, D., et al. 2006, A\&A, 454, 103

Henry, R. B. C., Kwitter, K. B., Jaskot, A. E., et al. 2010, ApJ, 724, 748

Hillier, D. J. 1991, A\&A, 247, 455

Hillier, D. J., \& Miller, D. L. 1998, ApJ, 496, 407

Hjorth, J., Sollerman, J., Møller, P., et al. 2003, Nature, 423, 847

Langer, N. 1992, A\&A, 265, L17

Lemasle, B., François, P., Bono, G., et al. 2007, A\&A, 467, 283

Levesque, E. M., Kewley, L. J., Graham, J. F., \& Fruchter, A. S. 2010, ApJ, 712 L26

Levesque, E. M., Berger, E., Soderberg, A. M., \& Chornock, R. 2011, ApJ, 739, 23

Maeder, A. 1987, A\&A, 178, 159

Mannucci, F., Salvaterra, R., \& Campisi, M. A. 2011, MNRAS, 414, 1263

Martayan, C., Frémat, Y., Hubert, A.-M., et al. 2006, A\&A, 452, 273

Martins, F., Hillier, D. J., Bouret, J. C., et al. 2009, A\&A, 495, 257

Massey, P., DeGioia-Eastwood, K., \& Waterhouse, E. 2001, AJ, 121, 1050

Meynet, G., \& Maeder, A. 2005, A\&A, 429, 581

Modjaz, M., Kewley, L., Kirshner, R. P., et al. 2008, AJ, 135, 1136

Mokiem, M. R., de Koter, A., Evans, C. J., et al. 2006, A\&A, 456, 1131

Mokiem, M. R., de Koter, A., Vink, J. S., et al. 2007, A\&A, 473, 603

Petrovic, J., Langer, N., \& van der Hucht, K. A. 2005a, A\&A, 435, 1013

Petrovic, J., Langer, N., Yoon, S.-C., \& Heger, A. 2005b, A\&A, 435, 247

Puls, J., Vink, J. S., \& Najarro, F. 2008, A\&A Rev., 16, 209

Sana, H., Gosset, E., \& Rauw, G. 2006, MNRAS, 371, 67

Sana, H., de Mink, S. E., de Koter, A., et al. 2012, Science, 337, 444

Sana, H., de Koter, A., de Mink, S. E., et al. 2013, A\&A, 550, A107

Thöne, C. C., Fynbo, J. P. U., Östlin, G., et al. 2008, ApJ, 676, 1151

van der Hucht, K. A. 2001, New Astron. Rev., 45, 135

Vink, J. S., de Koter, A., \& Lamers, H. J. G. L. M. 2001, A\&A, 369, 574

Vink, J. S., Gräfener, G., \& Harries, T. J. 2011, A\&A, 536, L10

Walborn, N. R., Morrell, N. I., Howarth, I. D., et al. 2004, ApJ, 608, 1028

Wellstein, S., Langer, N., \& Braun, H. 2001, A\&A, 369, 939

Woosley, S. E., Langer, N., \& Weaver, T. A. 1993, ApJ, 411, 823

Yoon, S.-C., Langer, N., \& Norman, C. 2006, A\&A, 460, 199 
F. Martins et al.: Quasi-homogeneous evolution of WN stars

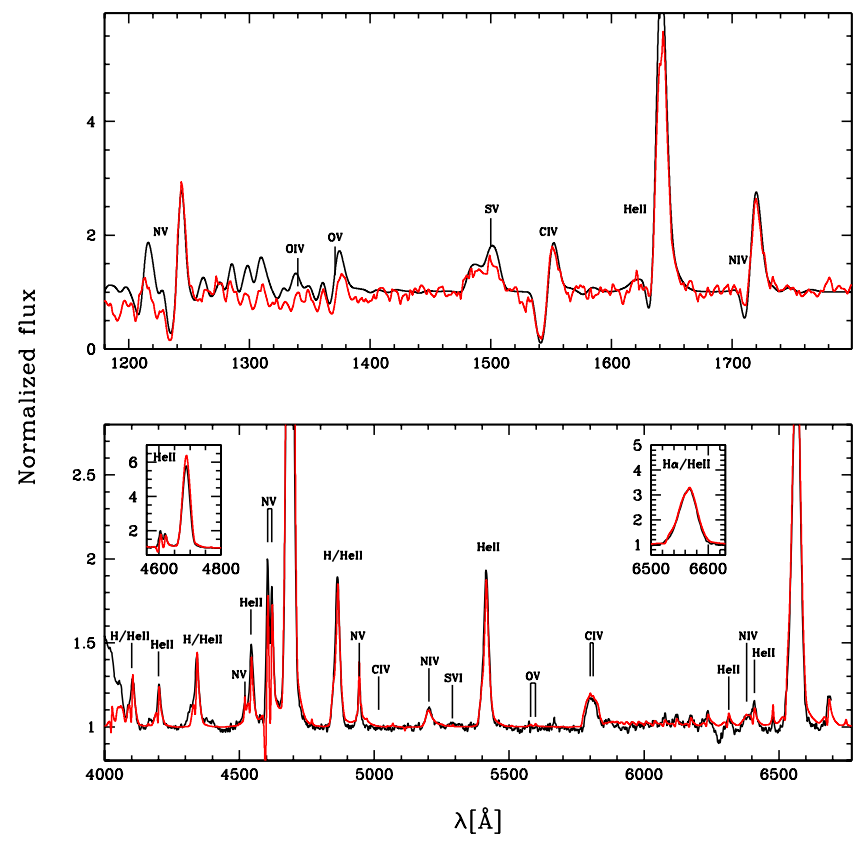

Fig. 1. CMFGEN model (red) compared to the observed spectrum (black) of star BAT 18.

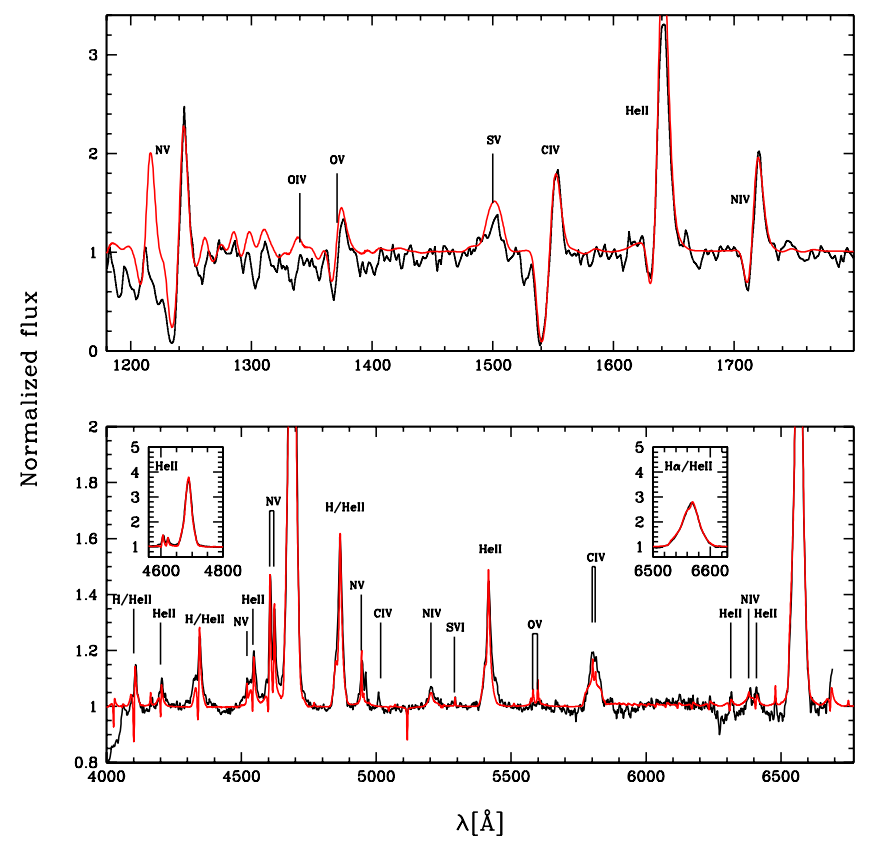

Fig. 2. CMFGEN model (red) compared to the observed spectrum (black) of star BAT 63.

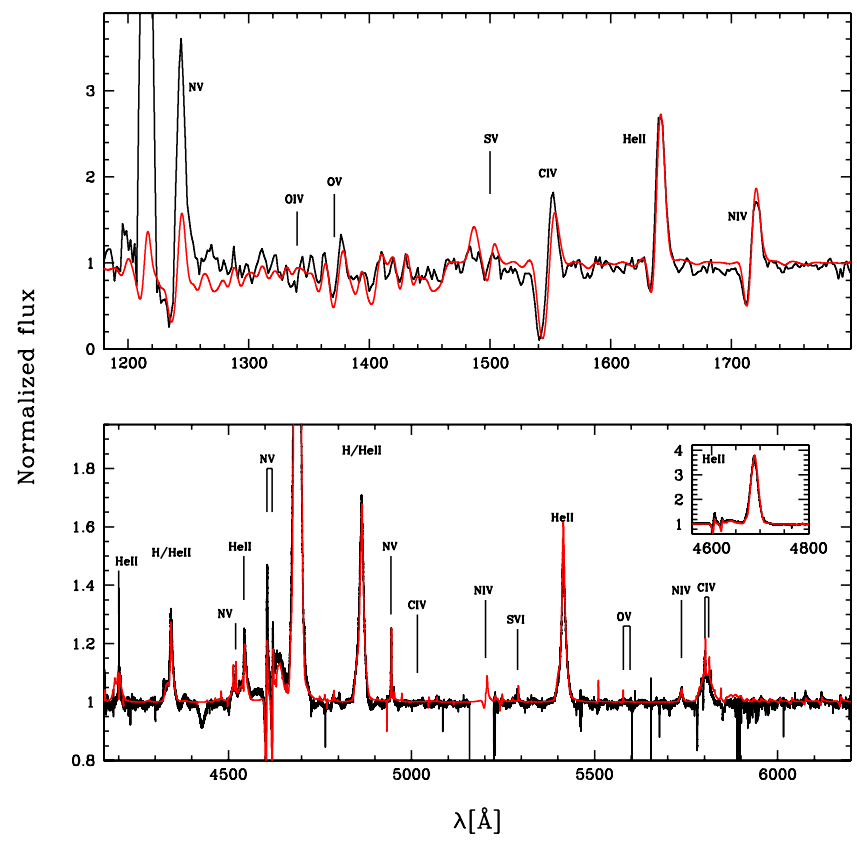

Fig. 3. CMFGEN model (red) compared to the observed spectrum (black) of star WR 10.

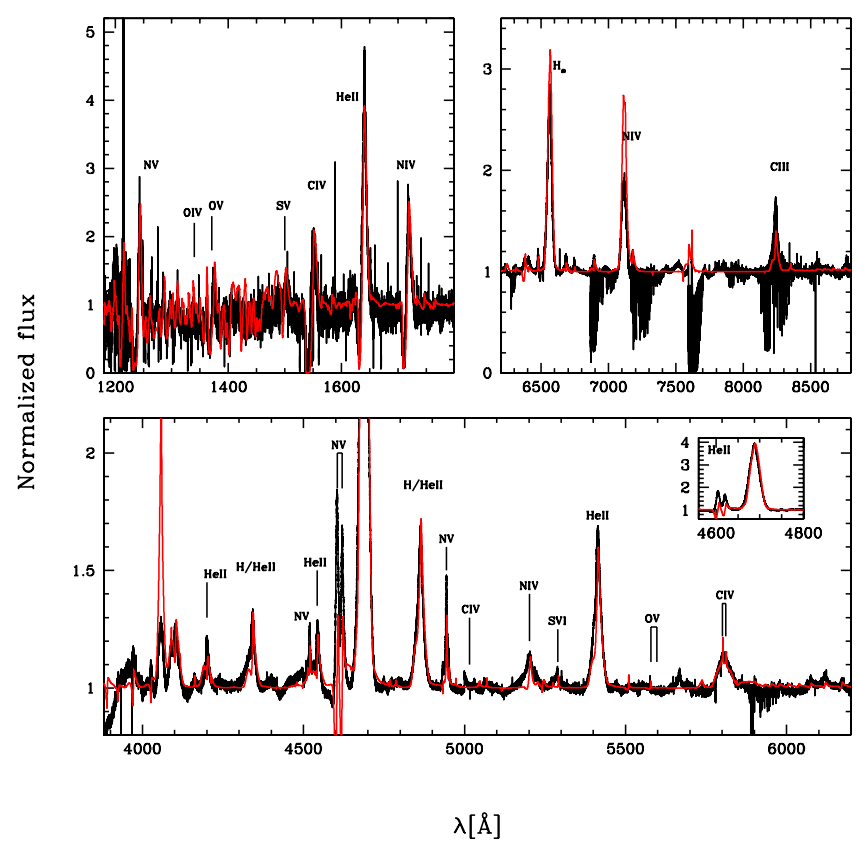

Fig. 4. CMFGEN model (red) compared to the observed spectrum (black) of star WR 128. 


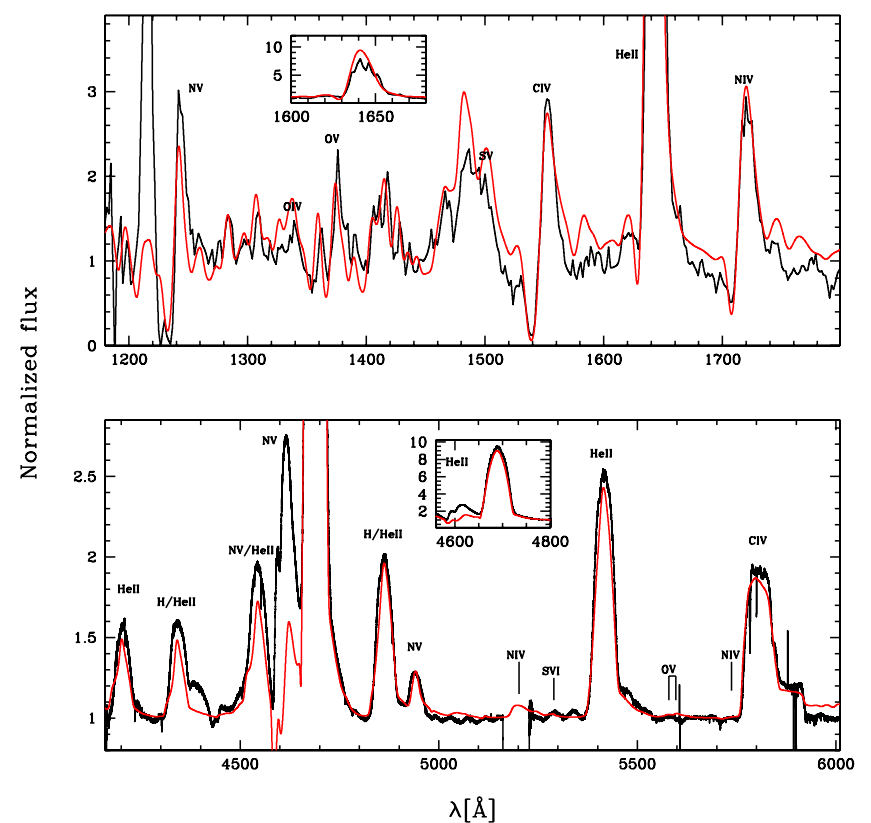

Fig. 5. CMFGEN model (red) compared to the observed spectrum (black) of star WR 18.

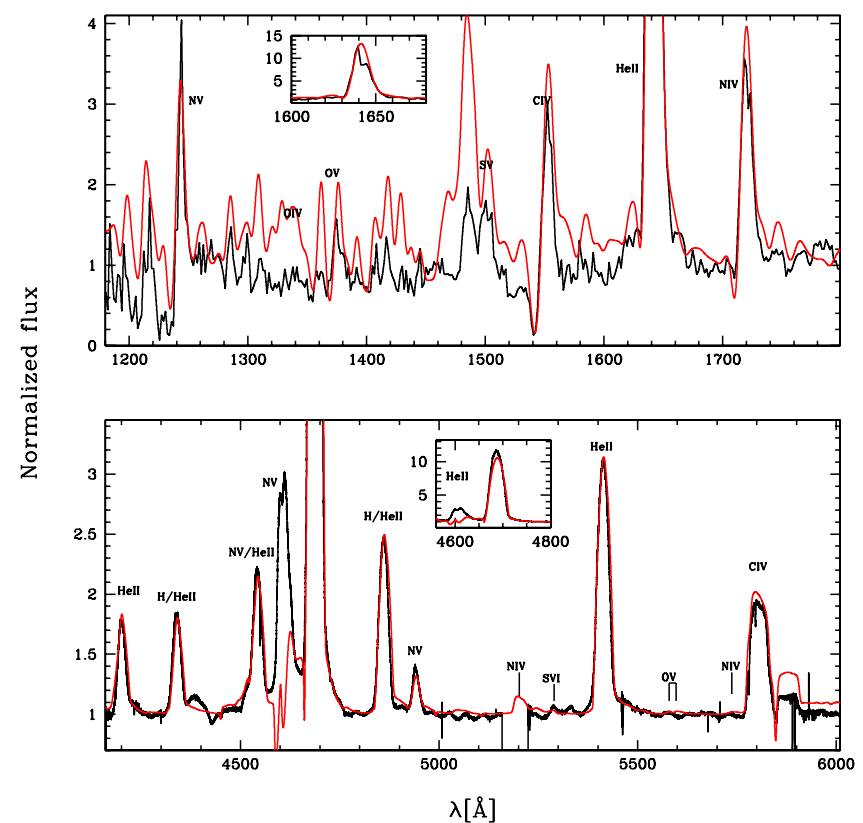

Fig. 6. CMFGEN model (red) compared to the observed spectrum (black) of star WR 7. 